Topic 3: Tidal Disruption Events 


\title{
Observational Progress in Identifying and Characterizing Tidal Disruption Flares
}

\author{
S. Bradley Cenko \\ Astrophysics Science Division, NASA Goddard Space Flight Center, Mail Code 661, 8800 \\ Greenbelt Road, Greenbelt, MD 20771, USA
}

\begin{abstract}
I present an overview of observational efforts across the electromagnetic spectrum to identify and study tidal disruption flares (TDFs), when a star wanders too close to a supermassive black hole and is torn apart by tidal forces. In particular I will focus on four unexpected surprises that challenge the most basic analytic picture of these events: 1) large inferred radii for the optical/UV-emitting material; 2) the ubiquity of outflows, detected at radio, X-ray, and UV wavelengths, ranging from speeds of $100 \mathrm{~km} / \mathrm{s}$ to near the speed of light; 3) the peculiar atomic abundances observed in the UV and optical spectra of these objects; and, 4) the preference for these events to occur in post-starburst galaxies.
\end{abstract}

Keywords. accretion, black hole physics

\section{Introduction}

When a star passes too close to a massive black hole, tidal forces will overcome self-gravity, unbinding the star. For Sun-like stars and sufficiently low-mass black holes $\left(M_{\mathrm{BH}} \lesssim 10^{8} \mathrm{M}_{\odot}\right)$, the disruption will occur outside the event horizon (Hills 1975). Approximately half $\dagger$ of the stellar debris will return to the black hole on highly elliptical orbits (Evans \& Kochanek 1989). The resulting emission from this returning bound debris is known as a tidal disruption flare (TDF; Rees 1988).

TDFs have gained increasing interest in the astronomical community in recent years, in large part due to their promise to address a number of key outstanding questions. First and foremost, TDFs provide a means to identify and probe super-massive black holes in distant galaxies that are otherwise inactive. As such, they may ultimately be used to extend $\mathrm{BH}$ mass measurements and the corresponding studies of black hole and galaxy evolution (e.g., the M-sigma relation; Ferrarese \& Merritt 2000; Gebhardt et al. 2000) well beyond the current reach of kinematic studies. In addition, TDFs serve as valuable probes of the process of accretion, providing insight into such fundamental questions as how accretion disks form, how disks behave at dramatically different Eddington ratios (e.g., De Colle et al. 2012), and how relativistic jets are generated in these systems (e.g., Levan et al. 2011; Bloom et al. 2011; Zauderer et al. 2011; Burrows et al. 2011; Cenko et al. 2012).

Early theoretical and numerical models of the tidal disruption process suggested that the resulting emission should be largely thermal in nature, with temperatures peaking at soft X-ray or extreme ultraviolet wavelengths (Ulmer 1999). Another early prediction was a characteristic $t^{-5 / 3}$ power-law decay for the mass return rate to pericenter (Phinney 1989), though it was not obvious that the observed emission in any one band would necessarily track the mass return rate (Strubbe \& Quataert 2009; Lodato \& Rossi 2011). The peak luminosity was anticipated to be roughly the Eddington luminosity for the corresponding black hole (e.g., $10^{44} \mathrm{erg} \mathrm{s}^{-1}$ for a $10^{6} \mathrm{M}_{\odot}$ black hole).

$\dagger$ The remainder is unbound and ejected from the system with high velocity. 
The earliest TDF discoveries were made largely in the X-ray band, with the ROSAT all-sky survey leading the way (see, e.g., Komossa 2015 for a review). These flares were identified largely by three properties: 1) large changes in amplitude (in some cases up to factors of thousands); 2) soft X-ray spectra (photon power-law indices $\gtrsim 4$, or blackbody spectra with $k T \gtrsim 50 \mathrm{eV}$ ); and, 3) coincident with the nucleus of a galaxy not previously known to be an AGN. The resulting luminosities and decay rates were largely consistent with early predictions; however it is worth noting that the sampling of these objects (both temporally in the X-rays and contemporaneous multi-wavelength observations) were relatively sparse. Since the completion of the ROSAT mission, TDFs have continued to be discovered at X-ray wavelengths though programs such as dedicated searches of galaxy clusters and via the wide-area coverage of the XMM Slew Survey (see, e.g., Saxton 2017 for an overview).

More recently, wide-field optical transient surveys such as the Palomar Transient Factory (Law et al. 2009; Rau et al. 2009), Pan-STARRS (Kaiser et al. 2010), and the All-Sky Automated Survey for SuperNovae (ASAS-SN; Shappee et al. 2014) have reinvigorated the field. While designed largely to discover supernovae by repeatedly imaging large areas of the sky on timescales of $\sim$ days, these surveys have now uncovered $\approx 10$ TDF candidates at optical wavelengths. And because these surveys identify their transient candidates in real-time, this has provided the opportunity for a broadband multi-wavelength perspective (e.g., optical spectroscopy, radio through X-ray imaging) on TDFs for the first time.

Not surprisingly, these optically discovered events have challenged our understanding of the tidal disruption process on a number of different fronts. Here I describe four recent "observational puzzles" that have arisen in recent years:

- Low and constant temperatures inferred from the optical/UV emission;

- Ubiquity of outflows across a broad range of velocity scales;

- Unusual abundance patterns in optical and UV spectra; and,

- A strong preference for post-starburst host galaxies.

Understanding all of these in the coming years will be critical to turning TDFs into viable probes of black hole mass in the upcoming era of the Large Synoptic Survey Telescope (LSST; Ivezic et al. 2008).

\section{Blackbody Temperature in Optical/UV TDFs}

As discussed above, the first TDF discoveries made at X-ray wavelengths had measured blackbody temperatures $T \lesssim 10^{6} \mathrm{~K}$. For a source with a peak luminosity $L \approx 10^{44} \mathrm{erg} \mathrm{s}^{-1}$, the implied blackbody radius is roughly comparable to the tidal radius (and several times the Schwarzchild radius for a $10^{6} \mathrm{M}_{\odot}$ black hole). These results are nicely consistent with models where the returning debris is able to circularize efficiently and form a radiatively efficient, optically thick (i.e., Shakura-Sunyaev) accretion disk.

However, nearly all TDFs discovered at optical and UV wavelengths exhibit very different spectral energy distributions. While the optical/UV emission is reasonably well characterized by a blackbody, the measured temperatures are significantly lower, typically $\approx 3 \times 10^{4} \mathrm{~K}$. As a result, the implied emission region is significantly larger than expected (i.e., much larger than the size anticipated for a newly formed accretion disk), with a characteristic size scale of $\approx 10^{15} \mathrm{~cm}$.

Even more puzzling, in most events the spectral temperature does not appear to vary significantly over the evolution of the flare, even though the luminosity may drop by orders of magnitude. Such evolution is dramatically different from supernovae, however, 
allowing for a powerful discriminant to identify TDFs amongst their much more numerous transient contaminants.

An illustrative example to consider is the nearby (and hence well-studied) ASASSN-14li $(d \approx 90 \mathrm{Mpc}$; Holoien et al. 2016; Van Velzen et al. 2016). ASASSN-14li is one of the few TDFs to exhibit both luminous optical/UV and X-ray emission, and they appear to derive from two physically distinct regions. The implied radius from fitting the $\mathrm{X}$-ray spectrum to a blackbody is consistent with the emission arising from the immediate environs of the tidal radius $\left(\approx 10^{13} \mathrm{~cm}\right)$. But the observed optical/UV emission is significantly more luminous than would be anticipated from extrapolating the X-ray spectrum to these wavelengths. Much like other optically discovered events, the spectral temperature and luminosity suggest a much larger emission region for the optical/UV emission.

Two competing models have been put forth to explain the small observed temperatures from optically discovered events. In the first, a "reprocessing" layer at large radii is responsible for reshaping the emission from the (inner) accretion disk, analogous to the photosphere in a supernova (Guillochon et al. 2014; Roth et al. 2016). The nature of this reprocessing layer remains somewhat mysterious, although it is natural to associate it with the outflowing material commonly seen in TDFs (see below). Varying the covering fraction and/or geometry of the reprocessing material can presumably alter the ratio of X-ray (accretion disk) to optical/UV (reprocessing) emission that is observed.

Alternatively, it may be that for some tidal disruptions the process of circularization (thought to be driven by stream-stream collisions) may be quite inefficient at generating an accretion disk (Piran et al. 2015; Krolik et al. 2016). Numerical simulations suggest that shocks may form very efficiently in these stream intersection regions, and thus it may be natural to expect emission directly from these locations (Shiokawa et al. 2015). While such a model would naturally explain the large inferred blackbody radii derived from optical/UV events, the wide range of observed X-ray-to-optical ratios in TDFs may be more challenging to accomodate.

\section{The Ubiquity of Outflows}

Accretion onto black holes, either in the guise of X-ray binaries (i.e., stellar mass black holes) or active galactic nuclei (i.e., super-massive black holes) can often give rise to outflows. The most dramatic example of these are the relativistic jets observed as microquasars and blazars, respectively. Given the similar physics involved in TDFs, it should not be surprising, then, to see analogous behavior.

It has been a bit surprising, however (to the author, at least) to see the large range of velocities over which outflows have been observed in only the handful of well-studied events to date. The most extreme examples are the newly formed relativistic jets that have been associated with tidal disruption events discovered by the Swift satellite: Sw J1644+57 (Levan et al. 2011; Bloom et al. 2011; Zauderer et al. 2011; Burrows et al. 2011), Sw J2058+05 (Cenko et al. 2012), and Sw J1112-82 (Brown et al. 2015). These sources are clearly very different beasts than the previously discussed tidal disruptions: extremely luminous ( $L \approx 10^{48} \mathrm{erg} \mathrm{s}^{-1}$ ) non-thermal X-ray emission is detected from these sources, lasting for roughly a year. Radio observations provide compelling evidence, mostly clearly in the case of Sw J1644+57, for a newly formed relativistic $\left(\Gamma_{0} \sim 2-10\right)$ jet powering these objects. Despite extensive searches (Bower et al. 2013; Van Velzen et al. 2013), only marginal evidence in one case (Irwin et al. 2015) has yielded potential emission from an off-axis jet, suggesting that this phenomena is relatively rare amongst the TDF population. 
While relativistic outflows may be relatively rare following the tidal disruption process, this does not suggest that all TDFs are radio quiet. As one of the most nearby events, ASASSN-14li once again stands as a valuable test case. Faint radio emission (orders of magnitude less luminous than Sw J1644+57) was detected from this source immediately following discovery (Alexander et al. 2016; Van Velzen et al. 2016). While the interpretation is somewhat complicated by the presence of a pre-outburst radio source, the most plausible interpretation appears to be that the radio emission is powered by an outflow with $v \approx 2 \times 10^{4} \mathrm{~km} \mathrm{~s}^{-1}$. A relatively modest fraction of energy is coupled to material moving with this speed $\left(\approx 10^{48} \mathrm{erg}\right)$, bringing to mind an analogy with supernovae and gamma-ray bursts (Alexander et al. 2016). Such outflows would likely be missed in more distant events due to their faintness.

Evidence for outflows with comparable velocity has also been observed from optical spectra of the TDF PS1-11af (Chornock et al. 2014). This source exhibited a strong absorption line in spectra near peak that seems most consistent with highly blueshifted $\left(\Delta v=-13,000 \mathrm{~km} \mathrm{~s}^{-1}\right) \mathrm{Mg}$ II $\lambda \lambda 2796,2803$. Interestingly, this absorption line is not present in early spectra of PS1-11af, possibly indicating that the observed outflow is associated with a specific accretion state (c.f., black hole X-ray binaries).

Perhaps most surprising of all, high-resolution X-ray spectroscopy of ASASSN-14li revealed a highly ionized, blueshifted absorption system with a remarkably low outflow speed: $\Delta v \approx-200 \mathrm{~km} \mathrm{~s}^{-1}$ (Miller et al. 2015). These absorption lines were observed to vary (both in strength and blueshift) on a time scale of a few days, indicating that they likely arose from very close to the black hole. However, the escape velocity at these small radii is significantly larger than the observed blueshift, raising a conundrum - how could such seemingly stable features arise from so close to the black hole?

Absorption lines from high-ionization material were also observed in the UV spectra of ASASSN-14li (Cenko et al. 2016). Given the similar measured blueshift, the simplest interpretation is that these lines arise from the same absorption seen in the X-rays. One potential clue to their origin may come from these UV spectra, which conspicuously lack common low-ionization absorption features such as Mg II and Fe II. Given the complex effects caused by photoionization in these systems (see below), one potential explanation is that we are directly observing stellar debris.

\section{Peculiar Abundance Measurements}

Optical spectra of the first optically selected TDF discovered in real-time, PS1-10jh, revealed a most peculiar abundance pattern: strong, broad emission lines of He II $\lambda 4686$ and $\lambda 3203$, but no corresponding Balmer emission lines. Given that the ionization energy of He II is significantly larger than that of H I, Gezari et al. (2012) argued that the lack of $\mathrm{H}$ in the spectra of PS1-10jh indicated a H-poor star was disrupted (see also Strubbe \& Murray 2015). If indeed the case, this would be a very surprising result, as He core stars are much rarer than typical main sequence (H-rich) stars. However, it would also imply that TDFs could be used as quite remarkable probes to study the chemical composition of individual stars in distant galaxies.

Subsequent to the discovery of PS1-10jh, a number of additional TDF candidates were identified by wide-field optical transient surveys, nearly all of which exhibited the characteristic broad He II emission lines (Arcavi et al. 2014). However, Balmer H emission (in particular $\mathrm{H} \alpha$ ) was observed in a number of these sources, and the H:He ratio has been observed to vary as a function of time even in the same source. A growing consensus appears to be emerging that photoionization effects play a pivotal role in the observed spectra of TDFs (Guillochon et al. 2014), and codes that make the common assumption 
in AGN environments that the gas is optically thin in the continuum are unlikely to be applicable in TDF environments. For instance, Roth et al. (2016) were able to match the observed $\mathrm{H}: \mathrm{He}$ ratio of $<1: 5$ in PS1-10jh using a H-rich star when properly solving for radiation transport.

The first UV spectrum of a TDF (ASASSN-14li) further reinforced the complicated radiation transport mechanisms in play in these systems (Cenko et al. 2016). Like AGN, broad nuclear emission lines are observed in the spectrum of ASASSN-14li. However, these lines display a remarkable abundance pattern: N III], N IV], and He II are quite prominent, while the common quasar emission lines of $\mathrm{C} \mathrm{III]} \mathrm{and} \mathrm{Mg}$ II are weak or entirely absent.

The lack of C III could be explained if the gas were above the critical density of $n_{\text {crit }}=10^{9.5} \mathrm{~cm}^{-3}$ (Osterbrock 1989); constraints from the X-ray spectrum place a lower limit of $n \gtrsim 2 \times 10^{9} \mathrm{~cm}^{-3}$. However, the strong $\mathrm{N}$ emission lines are reminiscent of a rare sub-class of quasars known as "N-rich" QSOs. Kochanek (2016) argues that these N-rich QSOs are in fact the tidal disruption of $\mathrm{a} \gtrsim 1 \mathrm{M}_{\odot}$ star, for which the high $\mathrm{N}$ abundance results from significant CNO processing in the stellar core (pre-disruption).

\section{A Preference for Post-Starburst Host Galaxies}

As has been the case for both gamma-ray bursts and all sub-classes of supernovae, the host galaxies of transients can often provide important diagnostics. In the case of tidal disruption flares, their hosts appear to exhibit a strong over-abundance of "poststarburst" galaxies: i.e., those galaxies with a strong red continuum from an old stellar population, but also exhibiting absorption lines of Balmer $\mathrm{H}$, indicating the presence of a recent "burst" of star-formation (Arcavi et al. 2014; French et al. 2016). Post-starburst galaxies are often invoked as relics of recent galaxy mergers. This has been suggested to explain their over-abundance, as galaxy mergers may alter the gravitational potential of the central black hole in a manner that rapidly fills its loss cone.

Understanding observational biases is important to make further progress in studies of TDF hosts. Most ground-based optical surveys select against nuclear transients in blue host galaxies, as these will suffer from significantly more contamination from AGN flares and core-collapse supernovae (potential TDF impostors). It is difficult to come up with a selection effect that would favor post-starburst galaxies over "ordinary" ellipticals; however, more detailed studies of rates are necessary to further understand this result.

\section{Future Prospects}

With convergence of both theoretical insight (see, e.g., Ramirez-Ruiz 2017 for an overview) and observational capabilities, TDFs will continue to experience exciting breakthroughs in the coming years. In the near-term, the advent of the Zwicky Transient Facility in 2017 should increase the optical TDF discovery rate by an order of magnitude, and eROSITA (Khabibullin et al. 2014) may uncover hundreds of TDFs at X-ray wavelengths (though less well-sampled).

However, there is still much work to be done to understand these remarkable transients. In the longer term, LSST is expected to uncover thousands of TDFs each year. But if these objects are to become viable probes of black hole mass in the distant universe, we will need a much better understanding of the physical processes at work in the interim. 


\section{References}

Alexander, K. D., Berger, E., Guillochon, J., Zauderer, B. A., \& Williams, P. K. G. 2016, ApJL, 819, L25

Arcavi, I., et al. 2014, ApJ, 793, 38

Bloom, J. S., et al. 2011, Science, 333, 203

Bower, G. C., Metzger, B. D., Cenko, S. B., Silverman, J. M., \& Bloom, J. S. 2013, ApJ, 763, 84

Brown, G. C., Levan, A. J., Stanway, E. R., Tanvir, N. R., Cenko, S. B., Berger, E., Chornock, R., \& Cucchiaria, A. 2015, MNRAS, 452, 4297

Burrows, D. N., et al. 2011, Nature, 476, 421

Cenko, S. B., et al. 2016, ApJL, 818, L32

-. 2012, ApJ, 753, 77

Chornock, R., et al. 2014, ApJ, 780, 44

De Colle, F., Guillochon, J., Naiman, J., \& Ramirez-Ruiz, E. 2012, ApJ, 760, 103

Evans, C. R. \& Kochanek, C. S. 1989, ApJL, 346, L13

Ferrarese, L. \& Merritt, D. 2000, ApJL, 539, L9

French, K. D., Arcavi, I., \& Zabludoff, A. 2016, ApJL, 818, L21

Gebhardt, K., et al. 2000, ApJL, 539, L13

Gezari, S., et al. 2012, Nature, 485, 217

Guillochon, J., Manukian, H., \& Ramirez-Ruiz, E. 2014, ApJ, 783, 23

Hills, J. G. 1975, Nature, 254, 295

Holoien, T. W.-S., et al. 2016, MNRAS, 455, 2918

Irwin, J. A., Henriksen, R. N., Krause, M., Wang, Q. D., Wiegert, T., Murphy, E. J., Heald, G., \& Perlman, E. 2015, ApJ, 809, 172

Ivezic, Z., et al. 2008, arXiv e-print (astro-ph/0805.2366)

Kaiser, N., et al. 2010, in Society of Photo-Optical Instrumentation Engineers (SPIE) Conference Series, Vol. 7733, Society of Photo-Optical Instrumentation Engineers (SPIE) Conference Series

Khabibullin, I., Sazonov, S., \& Sunyaev, R. 2014, MNRAS, 437, 327

Kochanek, C. S. 2016, MNRAS, 458, 127

Komossa, S. 2015, Journal of High Energy Astrophysics, 7, 148

Krolik, J., Piran, T., Svirski, G., \& Cheng, R. M. 2016, ApJ, 827, 127

Law, N. M., et al. 2009, PASP, 121, 1395

Levan, A. J., et al. 2011, Science, 333, 199

Lodato, G. \& Rossi, E. M. 2011, MNRAS, 410, 359

Miller, J. M., et al. 2015, Nature, 526, 542

Osterbrock, D. E. 1989, Astrophysics of gaseous nebulae and active galactic nuclei

Phinney, E. S. 1989, in IAU Symposium, Vol. 136, The Center of the Galaxy, ed. M. Morris, 543

Piran, T., Svirski, G., Krolik, J., Cheng, R. M., \& Shiokawa, H. 2015, ApJ, 806, 164

Rau, A., et al. 2009, PASP, 121, 1334

Rees, M. J. 1988, Nature, 333, 523

Roth, N., Kasen, D., Guillochon, J., \& Ramirez-Ruiz, E. 2016, ApJ, 827, 3

Shappee, B. J., et al. 2014, ApJ, 788, 48

Shiokawa, H., Krolik, J. H., Cheng, R. M., Piran, T., \& Noble, S. C. 2015, ApJ, 804, 85

Strubbe, L. E. \& Murray, N. 2015, MNRAS, 454, 2321

Strubbe, L. E. \& Quataert, E. 2009, MNRAS, 400, 2070

Ulmer, A. 1999, ApJ, 514, 180

van Velzen, S., et al. 2016, Science, 351, 62

van Velzen, S., Frail, D. A., Körding, E., \& Falcke, H. 2013, AAP, 552, A5

Zauderer, B. A., et al. 2011, Nature, 476, 425 